PROCEEDINGS OF THE

AMERICAN MATHEMATICAL SOCIETY

Volume 137, Number 10, October 2009, Pages 3187-3196

S 0002-9939(09)09975-4

Article electronically published on May 19, 2009

\title{
DISCREPANCY FOR RANDOMIZED RIEMANN SUMS
}

\author{
LUCA BRANDOLINI, WILLIAM CHEN, GIACOMO GIGANTE, \\ AND GIANCARLO TRAVAGLINI
}

(Communicated by Michael T. Lacey)

\begin{abstract}
Given a finite sequence $U_{N}=\left\{u_{1}, \ldots, u_{N}\right\}$ of points contained in the $d$-dimensional unit torus, we consider the $L^{2}$ discrepancy between the integral of a given function and the Riemann sums with respect to translations of $U_{N}$. We show that with positive probability, the $L^{2}$ discrepancy of other sequences close to $U_{N}$ in a certain sense preserves the order of decay of the discrepancy of $U_{N}$. We also study the role of the regularity of the given function.
\end{abstract}

\section{INTRODUCTION}

Let $N \in \mathbb{N}$ be a given large number, let $U_{N}=\left\{u_{1}, \ldots, u_{N}\right\}$ be a distribution of $N$ points in the unit cube $\left[-\frac{1}{2}, \frac{1}{2}\right)^{d}$, treated as the torus $\mathbb{T}^{d}$, and let $f$ be a real function on $\mathbb{T}^{d}$. Suppose that for suitable choices of $U_{N}$ and $f$, the Riemann sums

$$
\frac{1}{N} \sum_{j=1}^{N} f\left(u_{j}-x\right)
$$

are, after an $L^{2}$ average on the variable $x \in \mathbb{T}^{d}$, good approximations of the integral

$$
\int_{\mathbb{T}^{d}} f(s) \mathrm{d} s .
$$

What corresponding statement can we make concerning those sequences close to the sequence $U_{N}$ ? Do such sequences mostly share the same good behavior?

\section{A RANDOMIZATION ARGUMENT}

In order to start discussing these questions, we introduce the following randomization of $U_{N}$; see [3, 6] and also [8, 9]. Let $\mathrm{d} \mu$ denote a probability measure on $\mathbb{T}^{d}$. For every $j=1, \ldots, N$, let $\mathrm{d} \mu_{j}$ denote the measure obtained after translating $\mathrm{d} \mu$ by $u_{j}$. More precisely, for any integrable function $g$ on $\mathbb{T}^{d}$, we have

$$
\int_{\mathbb{T}^{d}} g(t) \mathrm{d} \mu_{j}=\int_{\mathbb{T}^{d}} g\left(t-u_{j}\right) \mathrm{d} \mu .
$$

Received by the editors June 24, 2008.

2000 Mathematics Subject Classification. Primary 11K38; Secondary 41A55.

Key words and phrases. Irregularities of distribution, decay of Fourier coefficients.

(C)2009 American Mathematical Society Reverts to public domain 28 years from publication 
Let $\mathrm{d} t$ denote the Lebesgue measure on $\mathbb{T}^{d}$. For every sequence $V_{N}=\left\{v_{1}, \ldots, v_{N}\right\}$ in $\mathbb{T}^{d}$ and every function $f \in L^{2}\left(\mathbb{T}^{d}, \mathrm{~d} t\right)$, we introduce, for every $t \in \mathbb{T}^{d}$, the discrepancy

$$
D\left(t, V_{N}\right) \stackrel{\text { def }}{=} \frac{1}{N} \sum_{j=1}^{N} f\left(v_{j}-t\right)-\int_{\mathbb{T}^{d}} f(s) \mathrm{d} s
$$

Observe that $D\left(\cdot, V_{N}\right)$ is a periodic function with Fourier series

$$
\sum_{0 \neq k \in \mathbb{Z}^{d}}\left(\frac{1}{N} \sum_{j=1}^{N} \mathrm{e}^{-2 \pi \mathrm{i} k \cdot v_{j}}\right) \overline{\widehat{f}(k)} \mathrm{e}^{2 \pi \mathrm{i} k \cdot t}
$$

and the Parseval identity yields

$$
D^{2}\left(V_{N}\right) \stackrel{\text { def }}{=}\left\|D\left(\cdot, V_{N}\right)\right\|_{L^{2}\left(\mathbb{T}^{d}, \mathrm{~d} t\right)}^{2}=\sum_{0 \neq k \in \mathbb{Z}^{d}}\left|\frac{1}{N} \sum_{j=1}^{N} \mathrm{e}^{2 \pi \mathrm{i} k \cdot v_{j}}\right|^{2}|\widehat{f}(k)|^{2}
$$

We now average $D\left(V_{N}\right)$ in $L^{2}\left(\mathbb{T}^{d}, \mathrm{~d} \mu_{j}\right)$ for every $j=1, \ldots, N$ and consider

$$
\mathfrak{D}_{\mathrm{d} \mu}\left(U_{N}\right) \stackrel{\text { def }}{=}\left(\int_{\mathbb{T}^{d}} \ldots \int_{\mathbb{T}^{d}} D^{2}\left(V_{N}\right) \mathrm{d} \mu_{1}\left(v_{1}\right) \ldots \mathrm{d} \mu_{N}\left(v_{N}\right)\right)^{1 / 2}
$$

In this paper we study the relation between $\mathfrak{D}_{\mathrm{d} \mu}\left(U_{N}\right)$ and $D\left(U_{N}\right)$. In the case $N=M^{d}$, where $M \in \mathbb{N}$, and

$$
U_{N}=\frac{1}{M} \mathbb{Z}^{d} \cap\left[-\frac{1}{2}, \frac{1}{2}\right)^{d}
$$

the above quantities were studied in relation to the sharpness of a result of Beck [1] and of Montgomery [10] on irregularities of distribution; see Remark 3 below. In [6] two of the authors compared the quantities $D\left(U_{N}\right)$ and $\mathfrak{D}_{\mathrm{d} \mu}\left(U_{N}\right)$ in the case (1) and when $f$ is the characteristic function of a ball. Here we study the problem in our more general setting, and we are mainly interested in whether the inequality

$$
\mathfrak{D}_{\mathrm{d} \mu}\left(U_{N}\right) \leqslant c D\left(U_{N}\right)
$$

holds.

Throughout this paper, the letters $c, C, \ldots$ will denote positive constants, possibly depending on $f$ but independent of $N$, and which may change from one step to the next. On the other hand, different letters $B, \kappa, \ldots$ will denote constants which will not change throughout the paper. 


\section{An EXPLiCIT FORMUla}

We first use a slight modification of an argument in [6] to obtain an explicit formula for $\mathfrak{D}_{\mathrm{d} \mu}\left(U_{N}\right)$. We have

$$
\begin{aligned}
& \mathfrak{D}_{\mathrm{d} \mu}^{2}\left(U_{N}\right) \\
& =\int_{\mathbb{T}^{d}} \ldots \int_{\mathbb{T}^{d}} \sum_{0 \neq k \in \mathbb{Z}^{d}}\left|\frac{1}{N} \sum_{j=1}^{N} \mathrm{e}^{2 \pi \mathrm{i} k \cdot v_{j}}\right|^{2}|\widehat{f}(k)|^{2} \mathrm{~d} \mu_{1}\left(v_{1}\right) \ldots \mathrm{d} \mu_{N}\left(v_{N}\right) \\
& =\sum_{0 \neq k \in \mathbb{Z}^{d}}|\widehat{f}(k)|^{2}\left(\frac{1}{N}+\frac{1}{N^{2}} \sum_{\substack{j, \ell=1 \\
j \neq \ell}}^{N} \int_{\mathbb{T}^{d}} \int_{\mathbb{T}^{d}} \mathrm{e}^{2 \pi \mathrm{i} k \cdot v_{j}} \mathrm{e}^{-2 \pi \mathrm{i} k \cdot v_{\ell}} \mathrm{d} \mu_{j}\left(v_{j}\right) \mathrm{d} \mu_{\ell}\left(v_{\ell}\right)\right) \\
& =\sum_{0 \neq k \in \mathbb{Z}^{d}}|\widehat{f}(k)|^{2} \\
& \times\left(\frac{1}{N}+\frac{1}{N^{2}} \sum_{\substack{j, \ell=1 \\
j \neq \ell}}^{N} \mathrm{e}^{2 \pi \mathrm{i} k \cdot\left(u_{\ell}-u_{j}\right)} \int_{\mathbb{T}^{d}} \int_{\mathbb{T}^{d}} \mathrm{e}^{2 \pi \mathrm{i} k \cdot v_{j}} \mathrm{e}^{-2 \pi \mathrm{i} k \cdot v_{\ell}} \mathrm{d} \mu\left(v_{j}\right) \mathrm{d} \mu\left(v_{\ell}\right)\right) \\
& =\sum_{0 \neq k \in \mathbb{Z}^{d}}|\widehat{f}(k)|^{2}\left(\frac{1}{N}+|\widehat{\mu}(k)|^{2}\left(\left|\frac{1}{N} \sum_{j=1}^{N} \mathrm{e}^{2 \pi \mathrm{i} k \cdot u_{j}}\right|^{2}-\frac{1}{N}\right)\right) \\
& =\frac{1}{N} \sum_{0 \neq k \in \mathbb{Z}^{d}}|\widehat{f}(k)|^{2}\left(1-|\widehat{\mu}(k)|^{2}\right)+\sum_{0 \neq k \in \mathbb{Z}^{d}}|\widehat{f}(k)|^{2}|\widehat{\mu}(k)|^{2}\left|\frac{1}{N} \sum_{j=1}^{N} \mathrm{e}^{2 \pi \mathrm{i} k \cdot u_{j}}\right|^{2} \\
& =\frac{1}{N}\left(\|f\|_{L^{2}\left(\mathbb{T}^{d}, \mathrm{~d} t\right)}^{2}-\|f * \mathrm{~d} \mu\|_{L^{2}\left(\mathbb{T}^{d}, \mathrm{~d} t\right)}^{2}\right)+\left\|D\left(\cdot, U_{N}\right) * \mathrm{~d} \mu\right\|_{L^{2}\left(\mathbb{T}^{d}, \mathrm{~d} t\right)}^{2} .
\end{aligned}
$$

There are two natural extremal measures. The first one is $\mathrm{d} \mu=\delta_{0}$, the Dirac measure centred at 0 . In this case, we have

$$
\mathfrak{D}_{\delta_{0}}\left(U_{N}\right)=D\left(U_{N}\right)
$$

On the other hand, when $\mathrm{d} \mu=\mathrm{d} t$, we have

$$
\mathfrak{D}_{\mathrm{d} t}^{2}\left(U_{N}\right)=\frac{1}{N}\left(\|f\|_{L^{2}\left(\mathbb{T}^{d}, \mathrm{~d} t\right)}^{2}-\left|\int_{\mathbb{T}^{d}} f(t) \mathrm{d} t\right|^{2}\right),
$$

the classical Monte-Carlo error.

Note that if $N D^{2}\left(U_{N}\right) \geqslant c$, then $\mathfrak{D}_{\mathrm{d} t}\left(U_{N}\right) \leqslant c_{1} D\left(U_{N}\right)$, and (2) follows easily.

Another very peculiar case is when $D\left(U_{N}\right)=0$. We observe that in general this does not imply $\mathfrak{D}_{\mathrm{d} \mu}\left(U_{N}\right)=0$, so that (2) does not hold. Indeed, let $U_{N}$ be given by (11). Then

$$
\frac{1}{N} \sum_{j=1}^{N} \mathrm{e}^{2 \pi \mathrm{i} k \cdot u_{j}}= \begin{cases}1 & \text { if } k \in M \mathbb{Z}^{d} \\ 0 & \text { otherwise. }\end{cases}
$$


Now choose $f(t)=\exp \left(2 \pi \mathrm{i} k_{0} \cdot t\right)$ for some $k_{0} \in \mathbb{Z}^{d} \backslash M \mathbb{Z}^{d}$. Then $D\left(U_{N}\right)=0$. On the other hand, it follows from (3) that

$$
\mathfrak{D}_{\mathrm{d} \mu}^{2}\left(U_{N}\right)=\frac{1}{N}\left(1-\left|\widehat{\mu}\left(k_{0}\right)\right|^{2}\right) \neq 0
$$

whenever $\left|\widehat{\mu}\left(k_{0}\right)\right| \neq 1$, which is easily fulfilled, particularly by several measures with small support around the origin.

Hence, throughout the paper, we will be interested only in the case when

$$
0<D\left(U_{N}\right)<N^{-1 / 2} .
$$

Let $0<\varepsilon_{N} \leqslant 1$. For every probability measure $\mathrm{d} \mu$ supported on the unit cube $\left[-\frac{1}{2}, \frac{1}{2}\right)^{d}$, let $\mathrm{d} \mu^{(N)}$ denote the probability measure defined by

$$
\int_{\mathbb{R}^{d}} g(\xi) \mathrm{d} \mu^{(N)}(\xi)=\int_{\mathbb{R}^{d}} g\left(\varepsilon_{N} \xi\right) \mathrm{d} \mu(\xi) .
$$

Then $\mathrm{d} \mu^{(N)}$ is supported on the subcube $\left[-\frac{1}{2} \varepsilon_{N}, \frac{1}{2} \varepsilon_{N}\right)^{d}$ and can be regarded as a measure on $\mathbb{T}^{d}$.

\section{MAin Result}

We first state our main result.

Theorem 1. Let $f \in L^{2}\left(\mathbb{T}^{d}, \mathrm{~d} t\right)$ and let $U_{N}=\left\{u_{1}, \ldots, u_{N}\right\}$ be a distribution of $N$ points in the cube $\left[-\frac{1}{2}, \frac{1}{2}\right)^{d}$. Assume that $0<D\left(U_{N}\right)<N^{-1 / 2}$. Let $\mathrm{d} \mu$ be a non-Dirac probability measure on $\mathbb{T}^{d}$, let $\mathrm{d} \mu^{(N)}$ be defined by (5) with $0<\varepsilon_{N} \leqslant 1$, and let

$$
\eta_{N}= \begin{cases}\varepsilon_{N}^{2 \alpha} & \text { if } \alpha<1, \\ \varepsilon_{N}^{2} \log \left(1+\varepsilon_{N}^{-1}\right) & \text { if } \alpha=1, \\ \varepsilon_{N}^{2} & \text { if } \alpha>1\end{cases}
$$

(i) If for some $\alpha>0$ and for every $\rho>1$ we have

$$
\sum_{\rho \leqslant|k|<2 \rho}|\widehat{f}(k)|^{2} \leqslant c \rho^{-2 \alpha},
$$

then

$$
\mathfrak{D}_{\mathrm{d} \mu^{(N)}}^{2}\left(U_{N}\right) \leqslant c \eta_{N} N^{-1}+D^{2}\left(U_{N}\right) .
$$

(ii) If there exists an open con 1$] \Omega \mathbb{R}^{d}$ such that for every subcone $\Gamma \subseteq \Omega$,

$$
\liminf _{\rho \rightarrow+\infty} \rho^{2 \alpha} \sum_{\substack{k \in \Gamma \\ \rho \leqslant|k|<2 \rho}}|\widehat{f}(k)|^{2}>0,
$$

then there exist positive constants $\Delta \leqslant 1$ and c such that if $\varepsilon_{N} \leqslant \Delta$, then

$$
\mathfrak{D}_{\mathrm{d} \mu(N)}^{2}\left(U_{N}\right) \geqslant c \eta_{N} N^{-1} \text {. }
$$

The following corollary shows that, in some sense, good sequences are never alone. Indeed we give conditions on $\varepsilon_{N}$ that will ensure that $\mathfrak{D}_{\mathrm{d} \mu^{(N)}}\left(U_{N}\right)$ and $D\left(U_{N}\right)$ are comparable.

Corollary 2. Let $f, U_{N}$ and $\mathrm{d} \mu$ be as given in Theorem 1 ,

\footnotetext{
${ }^{1}$ In this paper every cone starts from the origin.
} 
(i) Let $f$ be as given in part (i) of Theorem 1 and let

$$
\varepsilon_{N} \leqslant \begin{cases}\left(N^{1 / 2} D\left(U_{N}\right)\right)^{1 / \alpha} & \text { if } \alpha<1, \\ \beta_{N} & \text { if } \alpha=1, \\ N^{1 / 2} D\left(U_{N}\right) & \text { if } \alpha>1,\end{cases}
$$

where $\beta_{N}$ satisfies $\beta_{N}^{2} \log \left(1+\beta_{N}^{-1}\right)=N D^{2}\left(U_{N}\right)$. Then

$$
\mathfrak{D}_{\mathrm{d} \mu^{(N)}}^{2}\left(U_{N}\right) \leqslant c D^{2}\left(U_{N}\right) \text {. }
$$

(ii) Let $f$ and $\Delta$ be as given in part (ii) of Theorem 1 and let $\kappa>0$. Then there exists $c>0$ such that whenever

$$
\Delta \geqslant \varepsilon_{N} \geqslant \begin{cases}\kappa\left(N^{1 / 2} D\left(U_{N}\right)\right)^{1 / \alpha} & \text { if } \alpha<1 \\ \kappa \beta_{N} & \text { if } \alpha=1 \\ \kappa N^{1 / 2} D\left(U_{N}\right) & \text { if } \alpha>1\end{cases}
$$

we have

$$
\mathfrak{D}_{\mathrm{d} \mu^{(N)}}^{2}\left(U_{N}\right) \geqslant c D^{2}\left(U_{N}\right) .
$$

Remark 3. Consider the particular case when $f=\chi_{A}$, the characteristic function of a convex body $A \subseteq\left[-\frac{1}{2}, \frac{1}{2}\right)^{d}$. Then (6) holds with $\alpha=\frac{1}{2}$. Let $\varepsilon_{N}=\Delta N D^{2}\left(U_{N}\right)$. Then

$$
\mathfrak{D}_{\mathrm{d} \mu^{(N)}}^{2}\left(U_{N}\right) \leqslant c D^{2}\left(U_{N}\right) .
$$

If furthermore the boundary of $A$ is smooth and has positive Gaussian curvature, then (8) holds with $\alpha=\frac{1}{2}$; see, for instance, [7]. We then have

$$
\mathfrak{D}_{\mathrm{d} \mu^{(N)}}^{2}\left(U_{N}\right) \geqslant c D^{2}\left(U_{N}\right) .
$$

We recall that if $A$ is rotated and contracted, then a result of Beck [1] and of Montgomery [10 says that

$$
\int_{S O(d)} \int_{0}^{1} \int_{\mathbb{T}^{d}}\left|\frac{1}{N} \sum_{j=1}^{N} \chi_{\sigma(r A)}\left(u_{j}-t\right)-r^{d}\right| A||^{2} \mathrm{~d} t \mathrm{~d} r \mathrm{~d} \sigma \geqslant c N^{-1-1 / d}
$$

for every choice of the point set distribution $U_{N}$; see also [2, 4, 5]. We also recall that this is not true if the contraction is omitted; see [12, Theorem 3.1].

\section{DeCAy of the Fourier COefFicients}

The assumption (6) concerns the decay of the Fourier coefficients of $f$. This behavior can be naturally related to the smoothness of the function $f$ as follows. Let $f \in L^{2}\left(\mathbb{T}^{d}\right)$, define $\Delta_{h} f(x)=f(x+h)-f(x)$ and, for every integer $\ell \geqslant 1$, write $\Delta_{h}^{\ell} f=\Delta_{h} \Delta_{h}^{\ell-1} f$. Let $\alpha>0$. We say that $f$ belongs to the Nikol'skil space $H_{2}^{\alpha}\left(\mathbb{T}^{d}\right)$ if there exists $c>0$ such that

$$
\left(\int_{\mathbb{T}^{d}}\left|\Delta_{h}^{\ell} f(x)\right|^{2} \mathrm{~d} x\right)^{1 / 2} \leqslant c|h|^{\alpha}
$$

for some $\ell \geqslant 1$; see [11, Section 4.3.3].

Proposition 4. Let $f \in H_{2}^{\alpha}\left(\mathbb{T}^{d}\right)$. Then (6) holds. 
Proof. Since $\widehat{\Delta_{h} f}(k)=\left(\mathrm{e}^{2 \pi \mathrm{i} k \cdot h}-1\right) \widehat{f}(k)$, we have $\widehat{\Delta_{h}^{\ell}} f(k)=\left(\mathrm{e}^{2 \pi \mathrm{i} k \cdot h}-1\right)^{\ell} \widehat{f}(k)$. Let $h=(1 / 10 \rho, 0, \ldots, 0)$ and $\Gamma=\left\{k \in \mathbb{Z}^{d}: k_{1}^{2} \geqslant k_{2}^{2}+\ldots+k_{d}^{2}\right\}$. Observe that when $k \in \Gamma$ and $\rho \leqslant|k| \leqslant 2 \rho$, we have $\left|\mathrm{e}^{2 \pi \mathrm{i} k \cdot h}-1\right| \geqslant c$. Therefore

$$
\begin{aligned}
\sum_{\substack{k \in \Gamma \\
\rho \leqslant|k|<2 \rho}}|\widehat{f}(k)|^{2} \leqslant c \sum_{\substack{k \in \Gamma \\
\rho \leqslant|k|<2 \rho}}\left|\left(\mathrm{e}^{2 \pi \mathrm{i} k \cdot h}-1\right)^{\ell}\right|^{2}|\widehat{f}(k)|^{2} \leqslant c \sum_{k \in \mathbb{Z}^{d}}\left|\widehat{\Delta_{h}^{\ell} f}(k)\right|^{2} \\
=c \int_{\mathbb{T}^{d}}\left|\Delta_{h}^{\ell} f(x)\right|^{2} \mathrm{~d} x \leqslant c|h|^{2 \alpha}=c \rho^{-2 \alpha} .
\end{aligned}
$$

Note here that $h$ is tailored on $\Gamma$. Since we can cover $\mathbb{Z}^{d}$ with a finite number of cones, the proposition follows from the above argument applied to different choices of $h$.

The proof of Theorem 1 requires a technical lemma.

Lemma 5. Let $\mathrm{d} \nu$ be a probability measure supported on $\left[-\frac{1}{2}, \frac{1}{2}\right)^{d}$. Then either

(i) $\mathrm{d} \nu$ is the Dirac measure $\delta_{t_{0}}$ at a point $t_{0} \in\left[-\frac{1}{2}, \frac{1}{2}\right)^{d}$, or

(ii) $1-|\widehat{\nu}(\xi)|^{2}=O\left(|\xi|^{2}\right)$ as $\xi \rightarrow 0$, and any open cone in $\mathbb{R}^{d}$ contains an open subcone $\Gamma$ such that $1-|\widehat{\nu}(\xi)|^{2} \geqslant c|\xi|^{2}$ for small $\xi \in \Gamma$.

Proof. Since $\mathrm{d} \nu$ is compactly supported, its Fourier transform $\widehat{\nu}$ is smooth and has Taylor expansion

$$
\widehat{\nu}(\xi)=1+\nabla \widehat{\nu}(0) \cdot \xi+\frac{1}{2} H_{\widehat{\nu}}(0) \xi \cdot \xi+o\left(|\xi|^{2}\right),
$$

and so

$$
1-|\widehat{\nu}(\xi)|^{2}=1-\widehat{\nu}(\xi) \widehat{\nu}(-\xi)=(\nabla \widehat{\nu}(0) \cdot \xi)^{2}-H_{\widehat{\nu}}(0) \xi \cdot \xi+o\left(|\xi|^{2}\right)=O\left(|\xi|^{2}\right) .
$$

Let $F(\xi)=(\nabla \widehat{\nu}(0) \cdot \xi)^{2}-H_{\widehat{\nu}}(0) \xi \cdot \xi$, and assume that $F$ does not vanish identically. Let $\Sigma_{d-1}=\left\{\xi \in \mathbb{R}^{d}:|\xi|=1\right\}$. Since $F$ is a polynomial, it cannot vanish on an open set, and therefore $\left\{\xi \in \Sigma_{d-1}: F(\xi)=0\right\}$ has empty interior in $\Sigma_{d-1}$. Since $F$ is homogeneous and continuous, it follows that for every open cone in $\mathbb{R}^{d}$, we can find an open subcone $\Gamma$ such that $|F(\xi)| \geqslant c|\xi|^{2}$ for $\xi \in \Gamma$. Therefore $1-|\widehat{\nu}(\xi)|^{2} \geqslant c|\xi|^{2}$ for small $\xi \in \Gamma$.

Assume now that $F \equiv 0$. Observe that

$$
\frac{\partial \widehat{\nu}}{\partial \xi_{j}}(0)=-2 \pi \mathrm{i} \int_{\mathbb{T}^{d}} x_{j} \mathrm{~d} \nu(x)
$$

and

$$
\frac{\partial^{2} \widehat{\nu}}{\partial \xi_{j} \xi_{\ell}}(0)=-4 \pi^{2} \int_{\mathbb{T}^{d}} x_{j} x_{\ell} \mathrm{d} \nu(x)
$$

Then

$$
\nabla \widehat{\nu}(0) \cdot \xi=-2 \pi \mathrm{i} \int_{\mathbb{T}^{d}}(x \cdot \xi) \mathrm{d} \nu(x)
$$

and

$$
H_{\widehat{\nu}}(0) \xi \cdot \xi=-4 \pi^{2} \sum_{i, j} \int_{\mathbb{T}^{d}} \xi_{j} \xi_{\ell} x_{j} x_{\ell} \mathrm{d} \nu(x)=-4 \pi^{2} \int_{\mathbb{T}^{d}}(\xi \cdot x)^{2} \mathrm{~d} \nu(x) .
$$


Hence

$$
\begin{aligned}
0 & =(\nabla \widehat{\nu}(0) \cdot \xi)^{2}-H_{\widehat{\nu}}(0) \xi \cdot \xi=-4 \pi^{2}\left(\int_{\mathbb{T}^{d}}(x \cdot \xi) \mathrm{d} \nu(x)\right)^{2}+4 \pi^{2} \int_{\mathbb{T}^{d}}(\xi \cdot x)^{2} \mathrm{~d} \nu(x) \\
& =4 \pi^{2} \int_{\mathbb{T}^{d}}\left(x \cdot \xi-\int_{\mathbb{T}^{d}}(t \cdot \xi) \mathrm{d} \nu(t)\right)^{2} \mathrm{~d} \nu(x) .
\end{aligned}
$$

Let

$$
t_{0}=\int_{\mathbb{T}^{d}} t \mathrm{~d} \nu(t)
$$

Since $\mathrm{d} \nu(x)$ is positive, it follows that for every fixed $\xi$, we have

$$
\nu\left(\left\{x: x \cdot \xi-\xi \cdot t_{0} \neq 0\right\}\right)=0 .
$$

Since $\xi$ is arbitrary, we conclude that $\nu\left(\left\{x: x-t_{0} \neq 0\right\}\right)=0$, so that $\mathrm{d} \nu$ is supported at $t_{0}$. Since $\mathrm{d} \nu$ is a probability measure, we have $\mathrm{d} \nu=\delta_{t_{0}}$.

\section{Proof of Theorem 1}

By Lemma 5 , we have

$$
1-\left|\widehat{\mu^{(N)}}(k)\right|^{2}=1-\left|\widehat{\mu}\left(\varepsilon_{N} k\right)\right|^{2}=O\left(\varepsilon_{N}^{2}|k|^{2}\right) .
$$

As $\mathrm{d} \mu$ is a probability measure, we have

$$
0 \leqslant 1-\left|\widehat{\mu^{(N)}}(k)\right|^{2} \leqslant \min \left\{1, c \varepsilon_{N}^{2}|k|^{2}\right\} .
$$

By (6), we have

$$
\begin{aligned}
& \sum_{k \in \mathbb{Z}^{d}}|\widehat{f}(k)|^{2}\left(1-\left|\widehat{\mu^{(N)}}(k)\right|^{2}\right) \leqslant \sum_{k \in \mathbb{Z}^{d}}|\widehat{f}(k)|^{2} \min \left\{1, c \varepsilon_{N}^{2}|k|^{2}\right\} \\
& \leqslant \sum_{j=0}^{+\infty} \min \left\{1, c \varepsilon_{N}^{2} 2^{2 j}\right\} \sum_{2^{j} \leqslant|k|<2^{j+1}}|\widehat{f}(k)|^{2} \leqslant c \sum_{j=0}^{+\infty} \min \left\{1, \varepsilon_{N}^{2} 2^{2 j}\right\} 2^{-2 j \alpha} \\
& \quad \leqslant c \varepsilon_{N}^{2} \sum_{2^{j}<\varepsilon_{N}^{-1}} 2^{(2-2 \alpha) j}+c \sum_{2^{j}>\varepsilon_{N}^{-1}} 2^{-2 j \alpha} .
\end{aligned}
$$

There are three cases. If $\alpha<1$, we have

$$
\sum_{0 \neq k \in \mathbb{Z}^{d}}|\widehat{f}(k)|^{2}\left(1-\left|\widehat{\mu^{(N)}}(k)\right|^{2}\right) \leqslant c \varepsilon_{N}^{2 \alpha} .
$$

If $\alpha=1$, we have

$$
\sum_{0 \neq k \in \mathbb{Z}^{d}}|\widehat{f}(k)|^{2}\left(1-\left|\widehat{\mu^{(N)}}(k)\right|^{2}\right) \leqslant c \varepsilon_{N}^{2} \log \left(1+\varepsilon_{N}^{-1}\right) .
$$

If $\alpha>1$, we have

$$
\sum_{0 \neq k \in \mathbb{Z}^{d}}|\widehat{f}(k)|^{2}\left(1-\left|\widehat{\mu^{(N)}}(k)\right|^{2}\right) \leqslant c \varepsilon_{N}^{2} .
$$

Since $\mathrm{d} \mu$ is a probability measure, we have

$$
\left\|D\left(\cdot, U_{N}\right) * \mathrm{~d} \mu\right\|_{L^{2}\left(\mathbb{T}^{d}, \mathrm{~d} t\right)} \leqslant D\left(U_{N}\right) .
$$

In view of (11) and (12), the inequality (7) follows from (3). 
Let us now prove (ii). By Lemma 5 there exists a subcone $\Gamma \subset \Omega$ such that $1-|\widehat{\mu}(\xi)|^{2} \geqslant m_{1}|\xi|^{2}$ for $|\xi| \leqslant m_{2}, \xi \in \Gamma$. By (8) there exist $m_{3}$ and $m_{4}$ such that for $\rho \geqslant m_{3}$ we have

$$
\sum_{\substack{k \in \Gamma \\ \rho \leqslant|k|<2 \rho}}|\widehat{f}(k)|^{2} \geqslant m_{4} \rho^{-2 \alpha} .
$$

Thus, for $\varepsilon_{N}<\min \left\{m_{2} / 4 m_{3}, 1\right\}$, we have

$$
\begin{aligned}
\mathfrak{D}_{\mathrm{d} \mu^{(N)}}^{2}\left(U_{N}\right) & \geqslant \frac{1}{N}\left(\|f\|_{L^{2}\left(\mathbb{T}^{d}, \mathrm{~d} t\right)}^{2}-\left\|f * \mathrm{~d} \mu^{(N)}\right\|_{L^{2}\left(\mathbb{T}^{d}, \mathrm{~d} t\right)}^{2}\right) \\
& =\frac{1}{N} \sum_{0 \neq k \in \mathbb{Z}^{d}}|\widehat{f}(k)|^{2}\left(1-\left|\widehat{\mu}\left(\varepsilon_{N} k\right)\right|^{2}\right) \\
& \geqslant \frac{1}{N} \sum_{m_{3} \leqslant 2^{j} \leqslant \frac{1}{2} m_{2} \varepsilon_{N}^{-1}} \sum_{2^{j} \leqslant|k|<2^{j+1}}|\widehat{f}(k)|^{2}\left(1-\left|\widehat{\mu}\left(\varepsilon_{N} k\right)\right|^{2}\right) \\
& \geqslant \frac{\varepsilon_{N}^{2}}{N} m_{1} m_{4} \sum_{m_{3} \leqslant 2^{j} \leqslant \frac{1}{2} m_{2} \varepsilon_{N}^{-1}} 2^{-2 j \alpha} 2^{2 j} \geqslant c \eta_{N} N^{-1} .
\end{aligned}
$$

This completes the proof of Theorem 1

Remark 6. The estimates from below for $\mathfrak{D}_{\mathrm{d} \mu(N)}^{2}\left(U_{N}\right)$ contained in Theorem 1 and Corollary 2 depend on suitable estimates for the first term

$$
\frac{1}{N}\left(\|f\|_{L^{2}\left(\mathbb{T}^{d}, \mathrm{~d} t\right)}^{2}-\left\|f * \mathrm{~d} \mu^{(N)}\right\|_{L^{2}\left(\mathbb{T}^{d}, \mathrm{~d} t\right)}^{2}\right)
$$

in (3). We observe that in our setting the second term may vanish even in rather natural examples. Indeed, let

$$
f(x)=\sum_{k \neq 0} \frac{1}{|k| \gamma} \mathrm{e}^{2 \pi \mathrm{i} k x}
$$

for some $\gamma>d / 2+1$. One can easily check that (8) holds with $\alpha=\gamma-d / 2$. Let $U_{N}$ be as in (11) and $\mu$ be the (normalized) Lebesgue measure restricted to $\left[-\frac{1}{2}, \frac{1}{2}\right)^{d}$, so that, taking $\varepsilon_{N}=1 / M$, we have

$$
\widehat{\mu^{(N)}}(k)=N \prod_{j=1}^{d} \frac{\sin \left(\pi k_{j} / M\right)}{\pi k_{j}} .
$$

By (4) we have

$$
D^{2}\left(U_{N}\right)=\sum_{k \neq 0}|\widehat{f}(M k)|^{2}=\frac{1}{M^{2 \gamma}} \sum_{k \neq 0} \frac{1}{|k|^{2 \gamma}}=\frac{c_{\gamma}}{M^{2 \gamma}}
$$

and

$$
\left\|D\left(\cdot, U_{N}\right) * \mathrm{~d} \mu^{(N)}\right\|_{L^{2}\left(\mathbb{T}^{d}, \mathrm{~d} t\right)}=\sum_{k \neq 0}|\widehat{f}(M k)|^{2}\left|\widehat{\mu^{(N)}}(M k)\right|^{2}=0 .
$$

On the other hand observe that, for large $N$,

$$
\varepsilon_{N}=\frac{1}{M} \geqslant c_{\gamma} N^{\frac{1}{2}} D\left(U_{N}\right)=c_{\gamma} M^{d / 2-\gamma}
$$

and therefore we can apply part (ii) of Corollary 2 and obtain the inequality $\mathfrak{D}_{\mathrm{d} \mu^{(N)}}^{2}\left(U_{N}\right) \geqslant c D^{2}\left(U_{N}\right)$. 


\section{Conclusion}

Let $\mathrm{d} \mu^{\otimes}$ be defined on $\left(\mathbb{T}^{d}\right)^{N}$ by

$$
\int_{\left(\mathbb{T}^{d}\right)^{N}} \varphi \mathrm{d} \mu^{\otimes}=\int_{\mathbb{T}^{d}} \ldots \int_{\mathbb{T}^{d}} \varphi\left(v_{1}-u_{1}, \ldots, v_{N}-u_{N}\right) \mathrm{d} \mu^{(N)}\left(v_{1}\right) \ldots \mathrm{d} \mu^{(N)}\left(v_{N}\right) .
$$

We can now state and prove the result introduced in the abstract.

Corollary 7. Let $f, U_{N}$ and $\mathrm{d} \mu$ be as given in Corollary 2 .

(i) Let $f$ and $\varepsilon_{N}$ be as given in part (i) of Corollary 2, Then for every $\lambda$ satisfying $0<\lambda<1$, there exists a constant $c_{\lambda}>0$, independent of $U_{N}$ and such that $\mathrm{d} \mu^{\otimes}\left(\left\{V_{N}: D\left(V_{N}\right) \leqslant c_{\lambda} D\left(U_{N}\right)\right\}\right) \geqslant \lambda$.

(ii) Let $f, \Delta$ and $\varepsilon_{N}$ be as given in part (ii) of Corollary 2, Then for a suitable constant $c>0$, we have $\mathrm{d} \mu^{\otimes}\left(\left\{V_{N}: D\left(V_{N}\right) \geqslant c D\left(U_{N}\right)\right\}\right)>0$.

Proof. If (9) holds, then Corollary 2 gives

$$
\int_{\mathbb{T}^{d}} \cdots \int_{\mathbb{T}^{d}} D^{2}\left(V_{N}\right) \mathrm{d} \mu^{\otimes}\left(V_{N}\right) \leqslant c D^{2}\left(U_{N}\right)
$$

By the Chebyshev inequality, we have

$$
\mathrm{d} \mu^{\otimes}\left(\left\{V_{N}: D\left(V_{N}\right)>c_{\lambda} D\left(U_{N}\right)\right\}\right) \leqslant \frac{c}{c_{\lambda}^{2}},
$$

and so

$$
\mathrm{d} \mu^{\otimes}\left(\left\{V_{N}: D\left(V_{N}\right) \leqslant c_{\lambda} D\left(U_{N}\right)\right\}\right) \geqslant 1-\frac{c}{c_{\lambda}^{2}} .
$$

A suitable choice of $c_{\lambda}$ completes the proof of part (i). If (10) and (8) hold, then Corollary 2 gives

$$
\int_{\mathbb{T}^{d}} \cdots \int_{\mathbb{T}^{d}} D^{2}\left(V_{N}\right) \mathrm{d} \mu^{\otimes}\left(V_{N}\right) \geqslant c D^{2}\left(U_{N}\right)
$$

which easily implies $\mathrm{d} \mu^{\otimes}\left(\left\{V_{N}: D\left(V_{N}\right) \geqslant c D\left(U_{N}\right)\right\}\right)>0$.

\section{REFERENCES}

1. J. Beck, Irregularities of distribution. I, Acta Math. 159 (1987), 1-49. MR906524|(89c:11117)

2. J. Beck and W.W.L. Chen, Irregularities of Distribution, Cambridge Tracts in Mathematics, vol. 89, Cambridge University Press, 1987. MR903025 (88m:11061)

3. J. Beck and W.W.L. Chen, Note on irregularities of distribution. II, Proc. London Math. Soc. (3) 61 (1990), 251-272. MR.1063047 (91g:11083)

4. L. Brandolini, L. Colzani, and G. Travaglini, Average decay of Fourier transforms and integer points in polyhedra, Ark. Mat. 35 (1997), 253-275. MR 1478780 (99e:42021)

5. L. Brandolini, M. Rigoli, and G. Travaglini, Average decay of Fourier transforms and geometry of convex sets, Rev. Mat. Iberoamericana 14 (1998), 519-560. MR 1681584 (2000a:42017)

6. W.W.L. Chen and G. Travaglini, Deterministic and probabilistic discrepancies, Ark. Mat. (to appear).

7. C.S. Herz, Fourier transforms related to convex sets, Ann. of Math. (2) 75 (1962), 81-92. MR0142978 (26:545)

8. F.J. Hickernell and H. Woźniakowski, The price of pessimism for multidimensional quadrature, J. Complexity 17 (2001), 625-659. MR1881662 (2002m:60013)

9. D.P. Mitchell, Consequences of stratified samplings in graphics, SIGGRAPH 96: Proceedings of the 23rd Annual Conference on Computer Graphics and Interactive Techniques, Association for Computing Machinery, 1996, pp. 277-280.

10. H.L. Montgomery, Ten Lectures on the Interface between Analytic Number Theory and Harmonic Analysis, CBMS Regional Conference Series in Mathematics, vol. 84, Amer. Math. Soc., Providence, RI, 1994. MR,1297543 (96i:11002) 
11. S.M. Nikol'skiǔ, Approximation of Functions of Several Variables and Imbedding Theorems, Springer-Verlag, New York-Heidelberg, 1975. MR0374877(51:11073)

12. L. Parnovski and A.V. Sobolev, On the Bethe-Sommerfeld conjecture for the polyharmonic operator, Duke Math. J. 107 (2001), 209-238. MR1823047(2002d:35050)

Dipartimento di Ingegneria dell'Informazione e Metodi Matematici, Università di Bergamo, Viale Marconi 5, 24044 Dalmine, Bergamo, Italy

E-mail address: luca.brandolini@unibg.it

Department of Mathematics, Macquarie University, Sydney, NSW 2109, Australia

E-mail address: wchen@maths.mq.edu.au

Dipartimento di Ingegneria dell'Informazione e Metodi Matematici, Università di Bergamo, Viale Marconi 5, 24044 Dalmine, Bergamo, Italy

E-mail address: giacomo.gigante@unibg.it

Dipartimento di Statistica, Edificio U7, Università di Milano-Bicocca, Via Bicocca Degli Arcimboldi 8, 20126 Milano, Italy

E-mail address: giancarlo.travaglini@unimib.it 